Background: Multisystem inflammatory syndrome in children (MIS-C) is a rare complication of SARS-CoV-2 infection in the pediatric population, caused by extensive activation of immune system. The understanding of the distorted immune response is still in the early stages.

Objectives: To analyze comprehensively immune profile in MIS-C patients including detailed serologic response to SARS-CoV-2 in comparison with control groups.

Methods: Blood samples of consecutive MIS-C patients were collected at admission. Flow cytometric analysis of all lymphocyte populations including $T$ and $\mathrm{B}$ cell differentiation was performed. Immunophenotyping was performed by six-color panels for the detection of lymphocyte subpopulations. Anti-SARSCoV-2 specific antibodies were measured in the patients serum. The IgA and IgG antibodies against S protein, the IgG S1 and S2 specific antibodies, antibodies against nucleoprotein and neutralising antibodies were measured. Patients were assessed for a wide range of auto-antibodies, namely ANA, anti-ENA (Jo-1, PL-7, PL-12, SRP, Mi-2, Ku, Pm/Scl 100, Scl-70), myositis specific antibodies (EJ, MDA-5, TIH-Y, Ro52, SAE-1, SAE-2, NXP-2), anti-dsDNA, anti-phopholipid antibodies (aCl IgA, IgG, IgM, antiß2GPI IgG, IgM) and ANCA. Control groups to compare specific antibody response consisted of 14 healthy children and 19 healthy adults, who had SARS-CoV-2 infection in the last 2 months.

Results: Samples of 20 patients were included (14/20 boys, median age 12.4 years). Patients had higher percentage of double negative $T$ cells and low numbers of of cytokine producing T cells Th1, Th2 and Th17. . Numbers of immune competent and CD21+ transitional B cells were also lowered. All patients had positive antibodies against SARS-CoV-2 including neutralising antibodies. Nine $(9 / 19 ; 47 \%)$ patients had high titer $(\geq 1: 160)$ of neutralising antibodies. Results were compared with 2 control groups; 14 healthy children (7/14 boys; median age 8 years, ) and 19 healthy adults, who all experienced SARS-CoV-2 infection in the last two months. Patients with MIS-C had significantly higher levels of anti-S $\lg \mathrm{A}(\mathrm{p}<0.0001)$, patients with MIS-C and healthy children had significantly higher titers of anti-S1 $(p=0.001)$ and significantly lower titers of anti-S2 $(p=0.016)$ in comparison to adults (Figure 1). No differences were found in the titers of neutralising antibodies and anti-N antibodies. All patients were ANA negative, 19/20 patients were anti-ENA negative, whereas 1 patient had anti-Ro antibodies in low titre. Three patients had aCL IgG in medium titre and 2 patients anti-beta2GPI IgG in low titre. Patients were negative for all other autoantibodies.

Conclusion: The immune response in MIS-C patients is specific with most prominent differences in elevated percentage of double negative $T$ cells and low numbers of Th1, Th2, Th17 and CD21+ transitional B cells. MIS-C patients have distinct serologic response with high anti-S IgA, high anti-S1 and low anti-S2 titres.

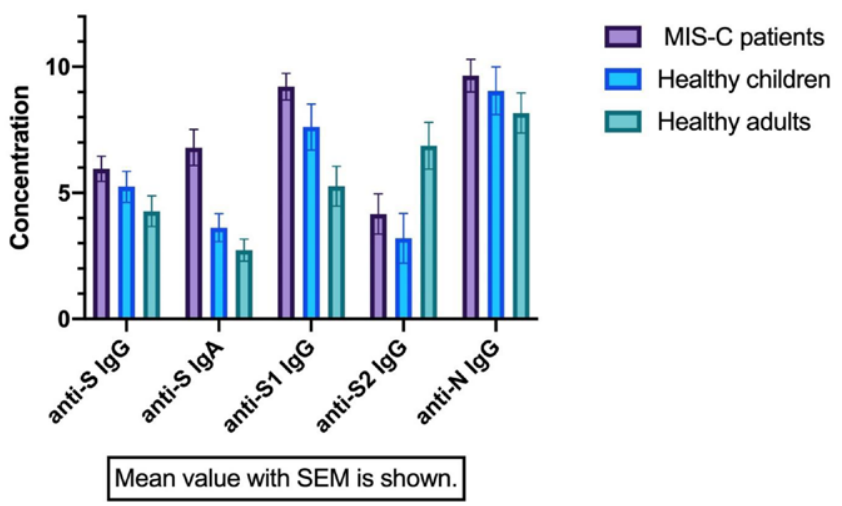

Figure 1. Antibody titres in patient group and control groups. Mean value with SEM s shown.

Disclosure of Interests: None declared.

DOI: 10.1136/annrheumdis-2021-eular.4109

\section{POS0073 1 ASSOCIATION OF SAA1 GENE POLYMORPHISM -13T/C (RS12218) WITH CRYOPYRIN ASSOCIATED PERIODIC SYNDROMES (CAPS), BUT NOT FAMILIAL MEDITERRANEAN FEVER (FMF)}

I. Guseva ${ }^{1}$, E. Fedorov ${ }^{2}$, S. Salugina ${ }^{2}$, M. Krylov ${ }^{1}$, E. Samarkina ${ }^{1}{ }^{1}$ V.A. Nasonova Research Institute of Rheumatology, Laboratory of Immunology and Molecular Biology of Rheumatic Diseases, Moscow, Russian Federation; ${ }^{2}$ V.A. Nasonova Research Institute of Rheumatology, Laboratory of Pediatric Rheumatic Diseases, Moscow, Russian Federation

Background: Autoinflammatory diseases (AIDs) are a group of rare, genetically determined diseases characterized by a periodic events of inflammation, fever, and clinical symptoms that mimic rheumatic pathology. Laboratory serological markers of AIDs are $C$ reactive protein (CRP) and serum amyloid $A$ (SAA) protein Objectives: We investigated whether $S A A 1$ gene polymorphism -13T/C (rs12218) may affect the susceptibility to pediatric FMF and CAPS patients (pts) We also evaluate whether this polymorphism can affect CRP and SAA protein levels.

Methods: 26 FMF pts - 8 boys, 18 girls; age - M(SD) 7.37(5.32) years and 24 CAPS pts - 12 girls, 12 boys; age $-4.76(5.83)$ years, and 95 healthy individuals (controls) were included in this study. The diagnosis of FMF was based on Turkish paediatric criteria for the diagnosis of familial Mediterranean fever [1] and was confirmed by the detection of pathogenic mutations of the MEFV gene in the homozygous or compound-heterozygous states. The diagnose of CAPS was made on the basis of characteristic clinical signs and was confirmed by the detection of a pathogenic mutation in the NLRP3 gene. SAA1 gene polymorphism -13T/C was genotyped using allele-specific RT-PCR assay. SAA protein concentration was measured using nephelometry in FMF and CAPS pts.

Results: Table 1 shows the genotypic and allelic frequencies of SSA1 gene in FMF, CAPS and controls. There were no significant differences between FMF pts and controls in the genotypic and allelic distributions of $-13 \mathrm{~T} / \mathrm{C}$ gene polymorphism. The frequency of $-13 \mathrm{C}$ allele was significantly higher in CAPS pts compared with controls ( $\mathrm{OR}=2.03[\mathrm{Cl} 1.02-4.03], \mathrm{p}=0.03$ ). Moreover, a statistically significant difference was revealed in the genotypic and allelic distributions between FMF and CAPS pts groups ( $p=0.047$ and $p=0.02$ respectively). The CRP levels did not correlate with SAA1 - 13T/C polymorphism. The SAA protein concentration was associated with $S A A 1$ gene polymorphism in FMF pts $(p=0.036)$.

Table 1. The distribution of genotypes and alleles of SAA1 gene polymorphism -13T/C in FMF, CAPS and control groups.

\begin{tabular}{lccc}
\hline Genotypes/Alleles & $\begin{array}{c}\text { FMF } \\
\mathrm{n}=26(\%)\end{array}$ & $\begin{array}{c}\text { CAPS } \\
\mathrm{n}=24(\%)\end{array}$ & $\begin{array}{c}\text { Controls } \\
\mathrm{n}=95(\%)\end{array}$ \\
\hline TT & $12(46,2)$ & $4(16,7)$ & $39(41,1)$ \\
TC & $12(46,2)$ & $14(58,3)$ & $42(44,2)$ \\
CC & $2(7,7)$ & $6(25,0)$ & $14(14,7)$ \\
P (pts vs controls) & $>0,05$ & $>0,08$ & $80(61,5)$ \\
T & $24(63,2)$ & $21(50,0)$ & $50(38,5)$ \\
C & $14(36,8)$ & $21(50,0)$ & \\
P (pts vs controls) & $>0,05$ & 0.03 & \\
\hline
\end{tabular}

Conclusion: Our preliminary study in small groups of pediatric FMF and CAPS pts revealed that SAA1 gene polymorphism -13T/C (rs12218) is associated with susceptibility to CAPS, but not FMF. The influence of this polymorphism on SAA protein levels in FMF pts was also shown. Further investigations are required to clarify the role of $S A A 1$ gene polymorphism $-13 T / C$ in susceptibility to FMF and CAPS in large studies in different ethnic and population groups.

\section{REFERENCES:}

[1] Yalcinkaya F, Ozen S, Ozcakar ZB, et al. Rheumatology (Oxford). 2009,48(4): 395-8. doi: 10.1093/rheumatology/ken509.

Disclosure of Interests: None declared.

DOI: 10.1136/annrheumdis-2021-eular.3910

\section{POS0074 CAN WE PREDICT THE DEVELOPMENT OF NEPHRITIS IN PEDIATRIC IGA VASCULITIS PATIENTS?}

E. Sag ${ }^{1,2}$, S. Demir ${ }^{1,2}$, Y. Bilginer ${ }^{1,2}$, S. Özen ${ }^{1,2} .{ }^{1}$ Hacettepe University Faculty of Medicine, Department of Pediatric Rheumatology, Ankara, Turkey; ${ }^{2}$ Hacettepe University Faculty of Medicine, Translational Medicine Laboratories, Pediatric Rheumatology Unit, Ankara, Turkey

Background: IgA vasculitis (IgAV) is the most common systemic vasculitis of childhood, characterized by palpable purpura, arthritis, gastrointestinal and rena involvement ${ }^{1}$. It is a relatively self-limited disease apart from the renal involvement which is associated with the long term morbidity.

Objectives: We aimed to define a marker at disease onset to predict the renal involvement.

Methods: In this pilot study, we analyzed a targeted panel of vascular inflam mation markers (sT2, RAGE, TIE-2, sCD40L, TIE-1, sFIt-1, LIGHT, TNF-a, PIGF IL6, IL18, IL10 and MCP-1) in the plasma samples of eight patients IgAV at the onset of the disease, before any treatment was initiated. At the time of sample collection, none of the patients had renal involvement; four of these patients subsequently developed nephritis and were defined as the IgAVN group. The levels of the markers were studied by a cytometric bead-based multiplex assay pane according to manufacturer's instruction (LEGENDplex HU Vascular Inflammation panel 2 (13-plex); catalogue number 740966, Biolegend) and analyzed by Novocyte 3005 flow cytometer. 